\title{
Identification of helpful and not helpful online reviews within an eWOM community using text-mining techniques
}

\author{
Olmedilla, Maria ${ }^{a}$; Martinez-Torres, Rocio ${ }^{b}$ and Toral, Sergio ${ }^{c}$ \\ a Research Center, Léonard de Vinci Pôle Universitaire, France, b Departmento \\ Administración de Empresas y Marketing, Universidad de Sevilla, Spain. ${ }^{\mathrm{c}}$ Departamento \\ de Ingeniería Electrónica, Universidad de Sevilla, Spain.
}

\begin{abstract}
Consumers represent today a significant source of information to learn about products and services quality thanks to the proliferation of user-generated content in the form of online reviews. It is thus of paramount to understand what makes online reviews helpful to consumers as this evaluation might affect their purchase decisions. In this regard, this research has applied textmining techniques by extracting the characteristics from online reviews' texts of an eWOM community, and further utilized these characteristics to train a logistic classifier using three classes: helpful, neutral and not helpful. The aim is identifying which unique attributes determine whether an online review is helpful or not. Findings reveal that there are much more unique attributes classified as helpful than attributes classified as not helpful. Additionally, the unique attributes associated to helpful reviews exhibit more objective appraisal while those associated to not helpful reviews show more subjective appraisal. The proposed methodology can be used to predict the helpfulness of posted reviews and to obtain their unique attributes.
\end{abstract}

Keywords: Text mining; unique attributes; objective and subjective appraisal; eWOM communities 